\title{
Relatively compatible operations in BCK-algebras and some related algebras
}

\author{
Lubomirsky N., San Martín H.J. and Zuluaga Botero W.J.
}

January 26, 2017

\begin{abstract}
Let $\mathbf{A}$ be a $B C K$-algebra and $f: A^{k} \rightarrow A$ a function. The main goal of this paper is to give a necessary and sufficient condition for $f$ to be compatible with respect to every relative congruence of $\mathbf{A}$. We extend this result in some related algebras, as for example in pocrims.
\end{abstract}

\section{Introduction and basic results}

If $\mathbf{A}$ is an algebra we write $A$ in order to refer to the universe of $\mathbf{A}$. Given a quasivariety $\mathcal{K}$ and $\mathbf{A} \in \mathcal{K}$ it is usually convenient to consider not every congruence of $\mathbf{A}$, but only those whose quotient $\mathbf{A} / \theta$ satisfies some additional conditions. It is natural to study only those congruences of $\mathbf{A}$ whose quotient $\mathbf{A} / \theta$ belongs to $\mathcal{K}$. If $\theta$ is a congruence in $\mathbf{A}$, we say that $\theta$ is a $\mathcal{K}$-congruence (or a congruence relative to $\mathcal{K}$ ) if $\mathbf{A} / \theta \in \mathcal{K}$. Let $\mathcal{K}$ be a quasivariety and $\mathbf{A} \in \mathcal{K}$. As usual, $\operatorname{Con}(\mathbf{A})$ denotes the partially ordered set of all congruences on $\mathbf{A}$ and $\operatorname{Con}_{\mathcal{K}}(\mathbf{A})$ denotes the partially ordered set of all $\mathcal{K}$-congruences on $\mathbf{A}$. It is well known that $\operatorname{Con}_{\mathcal{K}}(\mathbf{A})$ is an algebraic (complete) lattice [19]. For $\mathbf{A} \in \mathcal{K}, a \in A$ and $\theta$ an equivalence relation on $A$, we write $a / \theta$ for the equivalence class of $a$ associated to $\theta$. We also write $\theta(a, b)$ for the smallest congruence which contains the pair $(a, b)$ : these congruences are called principal congruences. Analogously, we write $\theta_{\mathcal{K}}(a, b)$ for the smallest $\mathcal{K}$-congruence containing the pair $(a, b)$, and these congruences are called principal $\mathcal{K}$-congruences.

Let $\mathcal{K}$ be a variety and $\mathbf{A} \in \mathcal{K}$. Then $\operatorname{Con}(\mathbf{A})=\operatorname{Con}_{\mathcal{K}}(\mathbf{A})$, so the definition of $\mathcal{K}$-congruence of $\mathbf{A}$ coincides with the definition of congruence of $\mathbf{A}$ and $\theta_{\mathcal{K}}(a, b)=\theta(a, b)$ for every $a, b \in A$.

Let $\mathbf{A}$ be an algebra and $f: A^{n} \rightarrow A$ a function. We say that $f$ is compatible with a congruence $\theta$ of $\mathbf{A}$ if $\left(a_{i}, b_{i}\right) \in \theta$ for $i=1 \ldots n$ implies $\left(f\left(a_{1}, \ldots, a_{n}\right), f\left(b_{1}, \ldots, b_{n}\right)\right) \in \theta$. We say that $f$ is a compatible operation of A provided it is compatible with all the congruences of $\mathbf{A}$. 
Definition 1.1. [7] Let $\mathcal{K}$ be a quasivariety, $\mathbf{A} \in \mathcal{K}$ and $f: A^{n} \rightarrow A$ a function. We say that $f$ is a $\mathcal{K}$-compatible operation of $\mathbf{A}$ provided it is compatible with all $\mathcal{K}$-congruences of $\mathbf{A}$.

If there is no ambiguity, we write relatively compatible operation instead of $\mathcal{K}$-compatible operation. Let $\mathbf{A}$ be an algebra, $f: A^{n} \rightarrow A$ a function and $a=\left(a_{1}, \ldots, a_{n}\right) \in A^{n}$. For $i=1, \ldots, n$ we define unary functions $f_{i}^{a}: A \rightarrow A$ by

$$
f_{i}^{a}(b):=f\left(a_{1}, \ldots, a_{i-1}, b, a_{i+1}, \ldots, a_{n}\right) .
$$

Remark 1.2. Let $\mathcal{K}$ be a quasivariety and $\mathbf{A} \in \mathcal{K}$.

1. Let $f: A \rightarrow A$ be a function. Then $f$ is $\mathcal{K}$-compatible if and only if $(f(a), f(b)) \in \theta_{\mathcal{K}}(a, b)$ for every $a, b \in A$.

2. Let $f: A^{n} \rightarrow A$ be a function. Then $f$ is $\mathcal{K}$-compatible if and only if for every $a \in A^{n}$ and every $i=1, \ldots, n$ the functions $f_{i}^{a}: A \rightarrow A$ are $\mathcal{K}$-compatible.

3. If $\mathcal{K}$ is a variety and $f: A^{n} \rightarrow A$ is a function then $f$ is $\mathcal{K}$-compatible if and only if $f$ is compatible.

In [12] it was introduced the implicit definibility by equations of new operations in quasivarieties.

Definition 1.3. [12] Let $\Sigma$ be a basis of a quasivariety $\mathcal{K}$ of type $\tau$. If $C$ is a set of new operations symbols such that $\tau \cap C=\emptyset$, and $E(C)$ is a set of identities in the extended type $\tau \cup C$, we denote by $\mathcal{K}(E(C))$ the quasivariety of algebras of type $\tau \cup C$ that satisfy the quasiequations in the set $\Sigma \cup E(C)$. We say that $E(C)$ implicitly defines $C$, if in each algebra $\mathbf{A} \in \mathcal{K}$ there is at most one family $\left\{f_{A}: A^{n} \rightarrow A\right\}_{f \in C}$ such that for every $f \in C$ we have that $\left(A, f_{A}\right) \in \mathcal{K}(E(C))$ and $f_{A}$ are $\mathcal{K}$-compatible. If $E(C)$ implicitly defines $C$ then we say that the quasivariety $\mathcal{K}(E(C))$ is a natural expansion of (the type $\tau$ of algebras in) $\mathcal{K}$.

In this paper we mainly consider $B C K$-algebras and related structures, like pocrims [11]. The variety of $B C K$ meet semilattices and the variety of $B C K$ join semilattices are natural expansions of the quasivariety of $B C K$ algebras, see [12, Subsection 4.2]. The quasivariety of pocrims is also a natural expansion of the quasivariety of $B C K$-algebras, which is mentioned in the last paragraph of [12, Section 1]. For more details about $B C K$ algebras see $[4,22,21,28]$.

Remark 1.4. Observe that for every $B C K$-algebra, the meet, the join and the fusion (product), if they exist, are relatively compatible operations. 
The classes of $B C K$ meet semilattices, pocrim meet semilattices and pocrim join semilattices are arithmetical varieties, so they are strictly locally affine complete varieties [26, Section 3.4.3]. References to fundamental papers on varieties of $B C K$-algebras and pocrims are $[4,5,25]$.

The paper is organized as follows. In Section 2 we bring a description of the relatively compatible operations in $B C K$-algebras. Moreover, we introduce an example of a new implicit function. In Section 3 we apply the results of Section 2 to the classes of pocrims [11], pocrim meet semilattices and pocrim join semilattices respectively. Finally, in Section 4 we generalize the obtained results to certain non-commutative analogues of $B C K$-algebras and pocrims, the so called pseudo $B C K$-algebras of the Rumanian school [27]. This section is mostly relating to managing the non-commutative aspect of the monoidal operation.

\section{Relatively compatible functions in BCK-algebras}

In this section we characterize the relatively compatible functions in $B C K$ algebras. We also introduce and study an example of relatively compatible function in $B C K$-algebras which is a generalization of the successor function studied by Caicedo and Cignoli as an example of an implicit compatible operation on Heyting algebras [6].

We start with some preliminary definitions and properties involving $B C K$-algebras and its logical counterpart. Furthermore, we describe the original motivation for the study of relatively compatible operations on $B C K$-algebras.

Definition 2.1. An algebra $(A, \rightarrow, 1)$ of type $(2,0)$ is a $B C K$-algebra if it satisfies the following conditions for every $a, b, c \in A$ :

$(\mathrm{BCK} 1)(a \rightarrow b) \rightarrow((b \rightarrow c) \rightarrow(a \rightarrow c))=1$,

$(\mathrm{BCK} 2) a \rightarrow((a \rightarrow b) \rightarrow b)=1$,

(BCK3) $a \rightarrow a=1$,

(BCK4) $a \rightarrow 1=1$,

(BCK5) if $a \rightarrow b=b \rightarrow a=1$ then $a=b$.

The above presentation of $B C K$-algebras is somewhat unusual. The most of the literature concerning $B C K$-algebras employs the dual notion, i.e., $a * b$ and 0 instead $b \rightarrow a$ and 1 respectively. We opt by the second notation since makes it possible to view the binary operation of $B C K$ algebras as a kind of implication and makes it more natural to add lattice operations to $B C K$-algebras as they correspond to the well known logical operations of conjunction and disjunction. The equivalence of this two forms 
of presentation is folklore (see for example [21]). More information about the subject can be found in [28].

In every $B C K$-algebra we can define a partial order by

$$
a \leq b \text { if and only if } a \rightarrow b=1 .
$$

The class of $B C K$-algebras is a quasivariety which is not a variety since, as it was proved in [32], it is not closed under homomorphic images. We write $\mathcal{B C K}$ for the quasivariety of $B C K$-algebras.

Remark 2.2. Let $\mathcal{K}$ be a quasivariety. We write $\mathbb{V}(\mathcal{K})$ for the variety generated by $\mathcal{K}$ and $\mathbb{H}(\mathcal{K})$ for the class of homomorphic images of members of $\mathcal{K}[31]$. Then $\mathbb{V}(K)=\mathbb{H}(K)$. Therefore, $\mathbb{V}(\mathcal{B C K})=\mathbb{H}(\mathcal{B C K})$.

We summarize some properties of $B C K$-algebras in the following lemma, whose proof can be found in [11].

Lemma 2.3. Let $\boldsymbol{A} \in \mathcal{B C K}$. Then the following conditions hold:

(1) $1 \rightarrow a=a$,

(2) $a \leq b$ implies $b \rightarrow c \leq a \rightarrow c$ and $c \rightarrow a \leq c \rightarrow b$,

(3) $a \leq b \rightarrow a$,

(4) $a \rightarrow(b \rightarrow c)=b \rightarrow(a \rightarrow c)$,

(5) $(a \rightarrow b) \rightarrow((c \rightarrow a) \rightarrow(c \rightarrow b))=1$,

(6) $a \rightarrow b=(((a \rightarrow b) \rightarrow b)) \rightarrow b$.

\subsection{Logical motivation}

The logic counterpart of the quasivariety $\mathcal{B C K}$ is the $\mathcal{B C K}$-logic, which is defined as follows [17]:

- The only connective in the language is $\rightarrow$.

- The axioms are three:

(B) $(\varphi \rightarrow \psi) \rightarrow((\psi \rightarrow \chi) \rightarrow(\varphi \rightarrow \chi))$,

(C) $(\varphi \rightarrow(\psi \rightarrow \chi)) \rightarrow(\psi \rightarrow(\varphi \rightarrow \chi))$,

(K) $\varphi \rightarrow(\psi \rightarrow \varphi)$.

- The only inference rule considered is Modus Ponens.

The preceding axioms imply the theorem $\varphi \rightarrow \varphi$. 
Remark 2.4. The $\mathcal{B C} \mathcal{K}$-logic is algebraizable with equivalence formulas $\{\varphi \rightarrow \psi, \psi \rightarrow \varphi\}$ and defining equation $\varphi \approx(\varphi \rightarrow \varphi)$. The quasivariety $\mathcal{B C K}$ is the equivalent algebraic semantics for the $\mathcal{B C K}$-logic [2].

Let $\mathrm{L}$ be an algebraizable logic [2] with a system of equivalence formulas $p \Leftrightarrow q$ (see [7] for details about notation). We write $\vdash_{\mathrm{L}} \nabla$ in order to indicate that $\nabla$ is deducible in $\mathrm{L}$. We say that an extension $\mathrm{L}(C)$ of $\mathrm{L}$ by axioms and rules involving a family of new connective symbols $C$ defines $C$ implicitly if $\vdash_{\mathrm{L}(C) \cup \mathrm{L}\left(C^{\prime}\right)} \nabla\left(p_{1} \ldots p_{n}\right) \Leftrightarrow \nabla^{\prime}\left(p_{1} \ldots p_{n}\right)$ for each $\nabla \in C$, where $C^{\prime}$ is a family of disjoint copies of the symbols in $C$, and $\mathrm{L}(C) \cup \mathrm{L}\left(C^{\prime}\right)$ is the structural extension of the logic $\mathrm{L}(C)$ by the $\nabla^{\prime}$-duplicates of the axioms and rules of $\mathrm{L}(C)$.

Remark 2.5. Let $\mathrm{L}$ be the $\mathcal{B C K}$-logic. Then $\mathrm{L}$ defines implicitly $C$ if $\vdash_{\mathrm{L}(C) \cup \mathrm{L}\left(C^{\prime}\right)} \nabla\left(p_{1} \ldots p_{n}\right) \rightarrow \nabla^{\prime}\left(p_{1} \ldots p_{n}\right)$ and $\vdash_{\mathrm{L}(C) \cup \mathrm{L}\left(C^{\prime}\right)} \nabla^{\prime}\left(p_{1} \ldots p_{n}\right) \rightarrow$ $\nabla\left(p_{1} \ldots p_{n}\right)$ for each $\nabla \in C$.

In algebraic logic, relatively compatible functions are concerned with implicitly defined connectives $[6,7]$. Adding connectives to extend a logic in a natural way has been broadly studied. For the classical propositional calculus, if an axiomatic extension defines implicitly a new connective it must be deductively equivalent to a combination of classical connectives. In the intuitionistic propositional calculus there are axiomatic extensions that define implicitly new connectives which are not deductively equivalent to a combination of intuitionistic connectives [6].

An extension of a logic $\mathrm{L}$ will be called axiomatic if it may be defined by adding a set of axiom schemes to L but no new inference rules. We will establish a link between the implicit connectives of an algebraizable logic $\mathrm{L}$ and the relatively compatible functions of the corresponding quasivariety $\mathcal{K}_{\mathrm{L}}$ obtained via the process of algebraization of Blok-Pigozzi [2].

Remark 2.6. Let $\mathrm{L}$ be an algebraizable logic and $\mathrm{L}(C)$ an axiomatic extension defining implicitly a family of connectives $C$. Then

1. $\mathrm{L}(C)$ is algebraizable via the same equivalence formulas and defining equations of $\mathrm{L}\left[7\right.$, Theorem 1]. Moreover, $\mathcal{K}_{\mathrm{L}(C)}$ consists of all algebras $\left(\mathbf{A}, \nabla_{\mathbf{A}}\right)_{\nabla \in C}, \mathbf{A} \in \mathcal{K}_{\mathrm{L}}$, satisfying the identities and quasi-identities corresponding to the axioms and rules of $\mathrm{L}(C)$. The algebraic interpretation of the connectives $\nabla \in C$ does not necessarily exist in every algebra $A \in \mathcal{K}_{\mathrm{L}}$. However, for each $\mathbf{A} \in \mathcal{K}_{\mathrm{L}}$ there is at most one family of functions $\left\{\nabla_{A}: \nabla \in C\right\}$ such that $\left(\mathbf{A}, \nabla_{\mathbf{A}}\right) \in \mathcal{K}_{\mathrm{L}}[7$, Corollary 2].

2. The functions $\nabla_{\mathbf{A}}, \nabla \in C$, are $\mathcal{K}_{\mathrm{L}}$-compatible whenever they exist [7, Theorem 4]. 
Remark 2.6 can be applied for $L=\mathcal{B C K}$. The logical motivation for the study of $\mathcal{B C K}$-compatible operations comes from the notion of implicit connective in the $\mathcal{B C} \mathcal{K}$-logic. However, in this paper we focus our attention only in the algebraic aspect of the subject. More precisely, we make an approach about $\mathcal{B C} \mathcal{K}$-compatible operations.

\section{$2.2 \quad \mathcal{B C K}$-compatible operations}

Let $\mathbf{A} \in \mathcal{B C K}$. Recall that a subset $F$ of $A$ is said to be an implicative filter (or deductive system) if $1 \in F$, and $b \in F$ whenever $a \in F$ and $a \rightarrow b \in F$ [12]. Implicative filters are also known as a (dual) ideal in the literature on $B C K$-algebras. In other words, the definition of implicative filter is another name for the familiar notion of (dual) ideal $[4,5]$. For any implicative filter $F$ we define the binary relation $\Theta_{F}$ by

$$
(a, b) \in \Theta_{F} \text { if and only if } a \rightarrow b \in F \text { and } b \rightarrow a \in F .
$$

In $[4$, Proposition 1] the following result is proved.

Lemma 2.7. Let $\boldsymbol{A} \in \mathcal{B C K}$. There exists an order isomorphism between the poset $\operatorname{Con}_{\mathcal{B C K}}(\boldsymbol{A})$ and the poset of implicative filters of $\boldsymbol{A}$, which is established via the functions $\theta \mapsto 1 / \theta$ and $F \mapsto \Theta_{F}$.

Corollary 2.8. Let $\boldsymbol{A} \in \mathcal{B C K}, a, b \in A$ and $\theta \in \operatorname{Con}_{\mathcal{B C K}}(\boldsymbol{A})$. Then $(a, b) \in \theta$ if and only if $(a \rightarrow b, 1) \in \theta$ and $(b \rightarrow a, 1) \in \theta$.

Proof. It follows from Lemma 2.7.

Let $\mathbf{A} \in \mathcal{B C K}$ and $a, b \in A$. We define $a \rightarrow^{1} b=a \rightarrow b$ and for $k \geq 1$,

$$
a \rightarrow^{k+1} b=a \rightarrow\left(a \rightarrow^{k} b\right)
$$

Let $X \subseteq A$. We write $\langle X\rangle$ by the filter generated by $X$. It is known that if $X \neq \emptyset$ then $\langle X\rangle$ is described by

$$
\langle X\rangle=\left\{b \in A: a_{1} \rightarrow\left(\ldots \rightarrow\left(a_{n} \rightarrow b\right) \ldots\right)=1 \text { for some } a_{1}, \ldots, a_{n} \in X\right\} .
$$

The previous description of $\langle X\rangle$ is due to Iséki $[23,24]$. In particular, if $X=\left\{a_{1}, \ldots a_{n}\right\}$ we write $\left\langle a_{1} \ldots a_{n}\right\rangle$ instead of $\left\langle\left\{a_{1} \ldots a_{n}\right\}\right\rangle$. For every $a \in A$ we have that

$$
\langle a\rangle=\left\{b \in A: a \rightarrow^{n} b=1 \text { for some } n\right\} .
$$

Let $\mathbf{A} \in \mathcal{B C K}$. The equations $a \rightarrow(b \rightarrow c)=b \rightarrow(a \rightarrow c)$ and $a \rightarrow 1=1$ imply that $a \in\langle b, c\rangle$ if and only if there are $n$ and $m$ natural numbers such that $b \rightarrow^{n}\left(c \rightarrow^{m} a\right)=1$, which is equivalent to say that there is a natural number $n$ such that $b \rightarrow^{n}\left(c \rightarrow^{n} a\right)=1$. 
Lemma 2.9. Let $\boldsymbol{A} \in \mathcal{B C K}$ and $a, b \in A$. Then $(c, d) \in \theta_{\mathcal{B C K}}(a, b)$ if and only if $c \rightarrow d \in\langle a \rightarrow b, b \rightarrow a\rangle$ and $d \rightarrow c \in\langle a \rightarrow b, b \rightarrow a\rangle$.

Proof. We write $\tau$ for arbitrary $\mathcal{B C K}$-congruences of $\mathbf{A}$. Since $\theta_{\mathcal{B C K}}(a, b)=$ $\bigcap_{(a, b) \in \tau} \tau$ then it follows from Corollary 2.8 that

$$
\theta_{\mathcal{B C K}}(a, b)=\bigcap_{a \rightarrow b, b \rightarrow a \in 1 / \tau} \tau .
$$

Then, by Lemma 2.7 we have that $1 / \theta_{\mathcal{B C K}}(a, b)=\langle a \rightarrow b, b \rightarrow a\rangle$. Thus, again by Corollary 2.8 we obtain $(c, d) \in \theta_{\mathcal{B C K}}(a, b)$ if and only if $c \rightarrow$ $d \in 1 / \theta_{\mathcal{B C K}}(a, b)$ and $d \rightarrow c \in 1 / \theta_{\mathcal{B C K}}(a, b)$, which happens if and only if $c \rightarrow d \in\langle a \rightarrow b, b \rightarrow a\rangle$ and $d \rightarrow c \in\langle a \rightarrow b, b \rightarrow a\rangle$.

In what follows we describe the unary $\mathcal{B C K}$-compatible functions:

Proposition 2.10. Let $\boldsymbol{A} \in \mathcal{B C K}$ and $f: A \rightarrow A$. Then $f$ is $\mathcal{B C K}$ compatible if and only if $f(a) \rightarrow f(b) \in\langle a \rightarrow b, b \rightarrow a\rangle$ for every $a, b \in A$.

Proof. It follows from Lemma 2.9.

The following corollary will be used in the next subsection.

Corollary 2.11. Let $\boldsymbol{A} \in \mathcal{B C K}$ and $f: A \rightarrow A$ a function.

(1) If $a \rightarrow b \leq(b \rightarrow a) \rightarrow(f(a) \rightarrow f(b))$ for every $a, b \in A$ then $f$ is $\mathcal{B C K}$-compatible.

(2) If $a \leq f(a)$ and $f(a \rightarrow b) \leq f(a) \rightarrow f(b)$ for every $a, b \in A$ then $f$ is $\mathcal{B C K}$-compatible.

Proof. Suppose that for every $a, b \in A, a \rightarrow b \leq(b \rightarrow a) \rightarrow(f(a) \rightarrow f(b))$. This inequality implies that $f(a) \rightarrow f(b) \in\langle a \rightarrow b, b \rightarrow a\rangle$. Hence, it follows from Proposition 2.10 that $f$ is $\mathcal{B C} \mathcal{K}$-compatible.

Finally suppose that for every $a, b \in A, a \leq f(a)$ and $f(a \rightarrow b) \leq f(a) \rightarrow$ $f(b)$. In particular, $a \rightarrow b \leq f(a \rightarrow b) \leq f(a) \rightarrow f(b)$. Since

$$
f(a) \rightarrow f(b) \leq(b \rightarrow a) \rightarrow(f(a) \rightarrow f(b))
$$

then $a \rightarrow b \leq(b \rightarrow a) \rightarrow(f(a) \rightarrow f(b))$. Therefore, $f$ is $\mathcal{B C K}$-compatible.

There are many connections between the existing literature and congruence preservation schemes [16] in the framework of $\mathcal{B C K}$-varieties, i.e., varieties contained in the quasivariety $\mathcal{B C K}$. In what follows we will make explicit some of these connections.

Let us begin by recalling that a $B C K$-algebra is said to be $(k+1)$ potent if $a \rightarrow^{k} b=a \rightarrow^{k+1} b$ for every $a, b[4,5]$. We write $\mathcal{E}_{k}$ for the class of $(k+1)$-potent $B C K$-algebras. 
Remark 2.12. The class $\mathcal{E}_{k}$ is a variety. Moreover, if $\mathbf{A} \in \mathcal{B C} \mathcal{K}$ and $A$ is finite then there is a natural number $k$ such that $\mathbf{A} \in \mathcal{E}_{k}[5]$.

A ternary deductive term (briefly, a TD term) for a variety $\mathcal{V}$ is a ternary term $\rho$ in the language of $\mathcal{V}$ such that: 1) $\mathcal{V}$ satisfies $\rho(a, a, c)=c ; 2$ ) for all $\mathbf{A} \in \mathcal{V}$ and $a, b, c, d \in A$, if $(c, d) \in \theta(a, b)$ then $\rho^{A}(a, b, c)=\rho^{A}(a, b, d)$.

Remark 2.13. The term $\rho(a, b, c)=(a \rightarrow b) \rightarrow^{k}\left((b \rightarrow a) \rightarrow^{k} c\right)$ is a TD term for the variety $\mathcal{E}_{k}[5]$.

It follows from [3, Corollary 2.5] that if $\mathcal{V}$ is a variety and $\mathbf{A} \in \mathcal{V}$ then $(c, d) \in \theta(a, b)$ if and only if $\rho(a, c, d)=\rho(b, c, d)$. So, combining the previous result with Remark 2.13 we obtain the following properties for $\mathbf{A} \in \mathcal{E}_{k}$ :

1) $(c, d) \in \theta(a, b)$ if and only if $(a \rightarrow b) \rightarrow^{k}\left((b \rightarrow a) \rightarrow^{k} c\right)=(a \rightarrow b) \rightarrow^{k}$ $\left((b \rightarrow a) \rightarrow^{k} d\right)$.

2) Let $f: A \rightarrow A$ be a function. Then $f$ is compatible if and only if $(a \rightarrow b) \rightarrow^{k}\left((b \rightarrow a) \rightarrow^{k} f(a)\right)=(a \rightarrow b) \rightarrow^{k}\left((b \rightarrow a) \rightarrow^{k} f(b)\right)$.

Hilbert algebras [14], also known as positive implicative $B C K$-algebras [13], may be characterized as the 2-potent $B C K$-algebras [5, pp. 294]. Thus, if $\mathbf{A}$ is a Hilbert algebra and $f: A \rightarrow A$ is a function then $f$ is compatible if and only if $(a \rightarrow b) \rightarrow((b \rightarrow a) \rightarrow f(a))=(a \rightarrow b) \rightarrow((b \rightarrow a) \rightarrow f(b))$ for every $a, b \in A$. Straightforward computations show that the last assertion is equivalent to the inequality $a \rightarrow b \leq(b \rightarrow a) \rightarrow(f(a) \rightarrow f(b))$ for every $a, b \in A$. This characterization of unary compatible functions in Hilbert algebras was also proved in [10].

\subsection{An example of $\mathcal{B C K}$-compatible operation: the successor}

During the decade of the 70's the Chisinau group headed by Kuznetsov introduced the notion of $\Delta$-pseudoboolean algebra. These algebras were later named KM-algebras by Esakia. Historical remarks about these algebras can be found in [29]. A KM-algebra is a Heyting algebra endowed with a unary function which satisfies certain identities. This unary function is called successor by Caicedo and Cignoli in [6]. They considered it as an example of an implicit compatible operation on Heyting algebras. The compatibility of the successor function was originally proved by Simonova in [31, Proposition 1].

Later, the successor function was generalized for the case of Hilbert algebras, and it was proved that it is also compatible [10]. Inspired by the definition given in [10], we will define a unary operation in $B C K$-algebras.

Definition 2.14. Let $\mathbf{A} \in \mathcal{B C K}$. A function $S: A \rightarrow A$ is called the successor function provided it satisfies the following inequalities: 
(S1) $a \leq S(a)$,

$(\mathrm{S} 2) \quad S(a) \leq((b \rightarrow a) \rightarrow b) \rightarrow b$,

(S3) $S(a) \rightarrow a=a$.

Remark 2.15. A function which satisfies these previous conditions is necessarily unique. In order to prove it, let $\hat{S}$ be a function that satisfies (S1), (S2) and (S3). It follows from $(\mathrm{S} 2)$ that $S(a) \leq((\hat{S}(a) \rightarrow a) \rightarrow \hat{S}(a)) \rightarrow \hat{S}(a)$. By (S3) we have that $\hat{S}(a) \rightarrow a=a$, so $S(a) \leq(a \rightarrow \hat{S}(a)) \rightarrow \hat{S}(a)$. Taking into account (S1) we have that $a \rightarrow \hat{S}(a)=1$, so $S(a) \leq 1 \rightarrow \hat{S}(a)=\hat{S}(a)$. Hence, $S(a) \leq \hat{S}(a)$. Replacing $S$ by $\hat{S}$ we obtain the other inequality. Therefore, $S(a)=\hat{S}(a)$. Also note that it follows from (S1) and (S3) that $S(x)=x$ if and only if $x=1$. In particular, $S(1)=1$.

We will call the successor function to the function $S$, or simply the successor. In [10], the successor for Hilbert algebras was defined through the inequalities (S2) and $S(a) \rightarrow a \leq S(a)$. In Hilbert algebras this definition is equivalent to the Definition 2.14, which follows from [10, Sect. 3, Corollary 4]. This property also holds for $B C K$-algebras.

Proposition 2.16. Let $\boldsymbol{A} \in \mathcal{B C K}$. The successor function is characterized by (S2) and the inequality $S(a) \rightarrow a \leq S(a)$.

Proof. The same proof of [10, Sect. 3, Lemma 3] can be applied.

Lemma 2.17. Let $\boldsymbol{A} \in \mathcal{B C K}$ and assume that the successor exists on $A$. Then

$$
S(a)=\min \{b \in A: b \rightarrow a=a \text { and } a \leq b\}
$$

for every $a \in A$.

Proof. For every $a \in A$ we define $E_{a}=\{b \in A: b \rightarrow a=a$ and $a \leq b\}$. By (S1) and (S3) we have that $S(a) \in E_{a}$. Let $b \in E_{a}$. Taking into account (S2) we have that

$$
\begin{aligned}
S(a) & \leq((b \rightarrow a) \rightarrow b) \rightarrow b \\
& =(a \rightarrow b) \rightarrow b \\
& =1 \rightarrow b \\
& =b .
\end{aligned}
$$

Therefore, $S(a) \leq b$, as needed.

In the following remark we will prove that it is possible to find a $B C K$ algebra with the following property: the function $\hat{S}$ given by $\hat{S}(a)=\min E_{a}$ exists but $\hat{S}$ is not the successor function. 
Remark 2.18. Consider the well known $M V$-algebra $H_{3}=\{0, a, 1\}$ with $0<a<1$. The implication is given by

\begin{tabular}{c|ccc}
$\rightarrow$ & 0 & $a$ & 1 \\
\hline 0 & 1 & 1 & 1 \\
$a$ & $a$ & 1 & 1 \\
1 & 0 & $a$ & 1
\end{tabular}

Then $\left(H_{3}, \rightarrow, 1\right) \in \mathcal{B C K}$. Straightforward calculations prove that $\hat{S}(b)=1$ for every $b \in H_{3}$. Since $((a \rightarrow 0) \rightarrow a) \rightarrow a=a$ and $\hat{S}(0) \not \leq a$ then (S2) is not satisfied by $\hat{S}$.

Lemma 2.19. Let $\boldsymbol{A} \in \mathcal{B C K}$ and assume that the successor exists on $A$. For every $a, b \in A$ the equality $(S(b) \rightarrow a) \rightarrow b=S(b) \rightarrow(a \rightarrow b)$ holds.

Proof. First we prove that

$$
a \rightarrow b=(S(b) \rightarrow a) \rightarrow b .
$$

In order to show this equality, let $a, b \in A$. Since $a \leq S(b) \rightarrow a$ then $a \rightarrow b \geq(S(b) \rightarrow a) \rightarrow b$. Conversely, $a \rightarrow b \leq(S(b) \rightarrow a) \rightarrow(S(b) \rightarrow b)$. Since $S(b) \rightarrow b=b$ then $a \rightarrow b \leq(S(b) \rightarrow a) \rightarrow b$. Thus we have proved (4). It follows from (4) that

$$
\begin{aligned}
(S(b) \rightarrow a) \rightarrow b & =a \rightarrow b \\
& =a \rightarrow(S(b) \rightarrow b) \\
& =S(b) \rightarrow(a \rightarrow b),
\end{aligned}
$$

which completes the proof.

Finally we will show the relatively compatibility of the successor.

Proposition 2.20. Let $\boldsymbol{A} \in \mathcal{B C K}$ and assume that the successor exists. Then, for every $a, b \in A, S(a \rightarrow b) \leq S(a) \rightarrow S(b)$. Moreover, $S$ is $\mathcal{B C K}$ compatible.

Proof. Let $a, b \in A$. By (S2) and Lemma 2.19 we have that

$$
\begin{aligned}
S(a \rightarrow b) & \leq((S(b) \rightarrow(a \rightarrow b)) \rightarrow S(b)) \rightarrow S(b) \\
& =((S(b) \rightarrow a) \rightarrow b) \rightarrow S(b)) \rightarrow S(b) .
\end{aligned}
$$

Then,

$$
S(a \rightarrow b) \leq((S(b) \rightarrow a) \rightarrow b) \rightarrow S(b)) \rightarrow S(b) .
$$

As $b \leq S(b)$ we have that $(S(b) \rightarrow a) \rightarrow b \leq(S(b) \rightarrow a) \rightarrow S(b)$. Thus,

$$
((S(b) \rightarrow a) \rightarrow S(b)) \rightarrow S(b) \leq((S(b) \rightarrow a) \rightarrow b) \rightarrow S(b) .
$$


Using (S2) and the inequality (6) we obtain that

$$
\begin{aligned}
S(a) & \leq((S(b) \rightarrow a) \rightarrow S(b)) \rightarrow S(b) \\
\leq & ((S(b) \rightarrow a) \rightarrow b) \rightarrow S(b),
\end{aligned}
$$

so we deduce the inequality

$$
((S(b) \rightarrow a) \rightarrow b) \rightarrow S(b)) \rightarrow S(b) \leq S(a) \rightarrow S(b) .
$$

It follows from (5) and (7) that

$$
S(a \rightarrow b) \leq S(a) \rightarrow S(b) .
$$

Therefore, it follows from the inequality (8) and Corollary 2.11 that $S$ is $\mathcal{B C K}$-compatible.

Remark 2.21. By Remark 2.15 and Proposition 2.20 we obtain that the quasivariety $\mathcal{B C K}(E(\{S\}))$ is a natural expansion of $\mathcal{B C K}$.

There are linearly-ordered Heyting algebras, i.e. Heyting algebras whose associated order is total, where it is not possible to define the successor function. For example, it is not possible to define the successor function in the real interval $[0,1]$ with the usual structure of Heyting algebra. In finite Heyting algebras there exists the successor function [15]. In particular, in finite linearly-ordered Heyting algebras we have that

$$
S(a)= \begin{cases}a^{+}, & \text {if } a \neq 1 \\ 1, & \text { if } a=1\end{cases}
$$

where $a^{+}$is the minimum of the set $\{b: b>a\}$.

Linearly-ordered $B C K$-algebras have been studied by García Olmedo and Rodríguez Salas [18] among others. There are finite linearly-ordered $B C K$-algebras where it is not possible to define the successor function, as it was mentioned in Remark 2.18. In the particular case of finite linearlyordered Hilbert-algebras with successor we have that $S(a)=a^{+}$for $a \neq$ 1. In order to show it, let $a \neq 1$. Suppose that $a^{+} \leq a^{+} \rightarrow a$. Then $a^{+} \rightarrow\left(a^{+} \rightarrow a\right)=1$. Since $a^{+} \rightarrow\left(a^{+} \rightarrow a\right)=\left(a^{+} \rightarrow a^{+}\right) \rightarrow\left(a^{+} \rightarrow a\right)$, $a^{+} \rightarrow a^{+}=1$ and $1 \rightarrow a^{+}=a^{+}$then $a^{+} \rightarrow a=1$. Thus we obtain $a^{+} \leq a$, which is a contradiction. Hence, $a^{+} \rightarrow a \leq a^{+}$. By (S2) we have that $S(a) \leq\left(\left(a^{+} \rightarrow a\right) \rightarrow a^{+}\right) \rightarrow a^{+}$, so $S(a) \leq a^{+}$. Then it follows from (S1) that $a \leq S(a) \leq a^{+}$, so $S(a)=a$ or $S(a)=a^{+}$. Suppose that $S(a)=a$, so $a=1$, which is a contradiction. Therefore, $S(a)=a^{+}$.

Based on the above discussion the following questions arise: 1) in finite linearly-ordered $B C K$-algebras where it is possible to define the successor function, does $S$ take the form $S(a)=a^{+}$for $a \neq 1$ ?; 2$)$ is $\mathcal{B C K}(E(\{S\}))$ a variety? 


\section{Relatively compatible operations in pocrims}

In this section we lift the results of the previous section to the quasivariety of pocrims. We start with some basic properties of pocrims which can be found in [11].

Definition 3.1. A structure $(A, \leq, \cdot, 1)$ is called a partially ordered monoid if $(A, \leq)$ is a poset, $(A, \cdot, 1)$ is a monoid and for all $a, b, c \in A$, if $a \leq b$ then $a \cdot c \leq b \cdot c$ and $c \cdot a \leq c \cdot b$. Moreover, if 1 is the greatest element of $(A, \leq)$ we say that $(A, \cdot, 1, \leq)$ is integral. A partially ordered commutative monoid is a partially ordered monoid where $\cdot$ is a commutative operation. A partially ordered commutative monoid $(A, \leq, \cdot, 1)$ is said to be residuated if for any $a, b \in A$ there is a largest $c \in A$, denoted by $a \rightarrow b$ and called the residuum of $a$ with respect to $b$, such that $a \cdot c \leq b$, i.e., $\rightarrow$ is an additional binary operation on $A$ satisfying the adjointness condition: $a \leq b \rightarrow c$ if and only if $a \cdot b \leq c$.

The term pocrim is an acronym for partially ordered commutative residuated integral monoid [11]. Since 1 is assumed to be the greatest element, the adjointness condition $a \leq b$ holds if and only if $a \rightarrow b=1$. This fact allows us to consider pocrims as algebras $(A, \cdot, \rightarrow, 1)$ of type $(2,2,0)$.

Pocrims constitute a quasivariety which is not a variety, that is, they are not closed under the formation of homomorphic images. This fact was proved by Higgs in [20]. In particular, we have that $\mathbb{V}(\mathcal{P O C})=\mathbb{H}(\mathcal{P O C})$.

Remark 3.2. An algebra $(A, \cdot, \rightarrow, 1)$ is a pocrim if and only if satisfies the following conditions: 1$)(A, \cdot, 1)$ is a commutative monoid; 2$)(A, \rightarrow, 1)$ is a $B C K$-algebra, 3) $(A, \cdot, \rightarrow, 1)$ satisfies the identity $(a \cdot b) \rightarrow c=a \rightarrow(b \rightarrow c)$. See for example [11, Theorem 1.7.8].

We write $\mathcal{P O C}$ for the class of pocrims. Let $(A, \cdot, \rightarrow, 1) \in \mathcal{P O C}$. Then $a \cdot(a \rightarrow b) \leq b$ and $a \rightarrow b \leq(a \cdot c) \rightarrow(b \cdot c)$. Let $A \in \mathcal{P O C}$ and $a \in A$. We define $a^{1}=a, a^{2}=a \cdot a$ and $a^{n+1}=a^{n} \cdot a$.

Remark 3.3. Let $\mathbf{A} \in \mathcal{P O C}$.

(1) If $F \subseteq A$ and $1 \in F$, we say that $F$ is a filter if $a \cdot b \in F$ whenever $a, b \in F$, and if $a \leq b$ and $a \in F$ then $b \in F$. In particular, $F$ is a filter if and only if $F$ is an implicative filter [11].

(2) If $F$ is a filter, then $a, b \in F$ if and only if $a \cdot b \in F$.

(3) If $X=\left\{a_{1}, \ldots, a_{n}\right\}$ then $\langle X\rangle=\left\langle a_{1} \cdot \ldots \cdot a_{n}\right\rangle$. Moreover, $\langle a\rangle=\{b \in A$ : $a^{n} \leq b$ for some natural number $\left.n\right\}$.

Let $\mathbf{A} \in \mathcal{P O C}$ and $a, b \in A$. We define the binary term $\leftrightarrow$ by $a \leftrightarrow b=$ $(a \rightarrow b) \cdot(b \rightarrow a)$. Straightforward calculations based in Corollary 2.8 and Remark 3.3 show the following lemma. 
Lemma 3.4. Let $\boldsymbol{A} \in \mathcal{P O C}, \theta \in \operatorname{Con}_{\mathcal{P O C}}(\boldsymbol{A})$ and $a, b \in A$. Then $(a, b) \in \theta$ if and only if $a \leftrightarrow b \in 1 / \theta$.

Lemma 3.5. Let $\boldsymbol{A} \in \mathcal{P O C}$ and $a, b \in A$. Then $(c, d) \in \theta_{\mathcal{P O C}}(a, b)$ if and only if $(a \leftrightarrow b)^{n} \leq c \leftrightarrow d$ for some natural number $n$.

Proof. It follows from Lemma 2.9 and Remark 3.3.

The following lemma follows from Lemma 3.5.

Lemma 3.6. Let $\boldsymbol{A} \in \mathcal{P O C}$ and $f: A \rightarrow A$ a function. Then $f$ is $\mathcal{P O C}$ compatible if and only if for every $a, b \in A$ there exists a natural number $n$ such that $(a \leftrightarrow b)^{n} \leq f(a) \leftrightarrow f(b)$.

In the following proposition we generalize Lemma 3.6.

Proposition 3.7. Let $\boldsymbol{A} \in \mathcal{P O C}$ and $f: A^{k} \rightarrow A$ a function.

The following conditions are equivalent:

(1) $f$ is $\mathcal{P O C}$-compatible.

(2) For every $a_{1}, \ldots, a_{k}, b_{1}, \ldots, b_{k} \in A$ there exists a natural number $n$ such that $\left(a_{1} \leftrightarrow b_{1}\right)^{n} \cdot \ldots \cdot\left(a_{k} \leftrightarrow b_{k}\right)^{n} \leq f\left(a_{1}, \ldots a_{k}\right) \leftrightarrow f\left(b_{1}, \ldots, b_{k}\right)$.

Proof. In this proof we will use that for $a, b, c \in A$ we have that

$$
(a \leftrightarrow b) \cdot(b \leftrightarrow c) \leq a \leftrightarrow c .
$$

Suppose that $f$ is $\mathcal{P O C}$-compatible and let $a_{1}, \ldots a_{k}, b_{1}, \ldots, b_{k} \in A$. By Remark 1.2 and Lemma 3.6 there are natural numbers $n_{1}, \ldots, n_{k}$ such that

$$
\begin{aligned}
& \left(a_{1} \leftrightarrow b_{1}\right)^{n_{1}} \leq f\left(a_{1}, a_{2}, \ldots, a_{k}\right) \leftrightarrow f\left(b_{1}, a_{2}, \ldots, a_{k}\right), \\
& \left(a_{2} \leftrightarrow b_{2}\right)^{n_{2}} \leq f\left(b_{1}, a_{2}, a_{3}, \ldots, a_{k}\right) \leftrightarrow f\left(b_{1}, b_{2}, a_{3}, \ldots, a_{k}\right), \\
& \vdots \\
& \left(a_{k} \leftrightarrow b_{k}\right)^{n_{k}} \leq f\left(b_{1}, \ldots, b_{k-1}, a_{k}\right) \leftrightarrow f\left(b_{1}, b_{2}, \ldots, b_{k}\right) .
\end{aligned}
$$

Let $n$ be the maximum of the set $\left\{n_{1}, \ldots, n_{k}\right\}$. Then, for every $i=1, \ldots, k$ we obtain that $\left(a_{i} \leftrightarrow b_{i}\right)^{n} \leq\left(a_{i} \leftrightarrow b_{i}\right)^{n_{i}}$. Thus,

$$
\begin{aligned}
& \left(a_{1} \leftrightarrow b_{1}\right)^{n} \leq f\left(a_{1}, a_{2}, \ldots, a_{k}\right) \leftrightarrow f\left(b_{1}, a_{2}, \ldots, a_{k}\right), \\
& \left(a_{2} \leftrightarrow b_{2}\right)^{n} \leq f\left(b_{1}, a_{2}, a_{3}, \ldots, a_{k}\right) \leftrightarrow f\left(b_{1}, b_{2}, a_{3}, \ldots, a_{k}\right), \\
& \vdots \\
& \left(a_{k} \leftrightarrow b_{k}\right)^{n} \leq f\left(b_{1}, \ldots, b_{k-1}, a_{k}\right) \leftrightarrow f\left(b_{1}, b_{2}, \ldots, b_{k}\right) .
\end{aligned}
$$

Hence, straightforward computations based in (9) show that

$$
\left(a_{1} \leftrightarrow b_{1}\right)^{n} \cdot \ldots \cdot\left(a_{k} \leftrightarrow b_{k}\right)^{n} \leq f\left(a_{1}, \ldots a_{k}\right) \leftrightarrow f\left(b_{1}, \ldots, b_{k}\right) .
$$


Conversely, assume the condition (2). Let $\theta \in \operatorname{Con}_{\mathcal{P O C}}(\mathbf{A})$ and $\left(a_{i}, b_{i}\right) \in$ $\theta$ for $i=1 \ldots, k$. We will prove that $\left(f\left(a_{1}, \ldots, a_{k}\right), f\left(b_{1}, \ldots, b_{k}\right)\right) \in \theta$. In order to show it, first note that condition (2) says that there is a natural number $n$ such that

$$
\left(a_{1} \leftrightarrow b_{1}\right)^{n} \cdot \ldots \cdot\left(a_{k} \leftrightarrow b_{k}\right)^{n} \leq f\left(a_{1}, \ldots a_{k}\right) \leftrightarrow f\left(b_{1}, \ldots, b_{k}\right) .
$$

Since $\left(a_{i}, b_{i}\right) \in \theta$ then it follows from Lemma 3.4 that $\left(a_{i} \leftrightarrow b_{i}, 1\right) \in \theta$, so $\left(\left(a_{i} \leftrightarrow b_{i}\right)^{n}, 1\right) \in \theta$. Since $\left(a_{i} \leftrightarrow b_{i}\right)^{n} \in 1 / \theta$ then

$$
\left(a_{1} \leftrightarrow b_{1}\right)^{n} \cdot \ldots \cdot\left(a_{k} \leftrightarrow b_{k}\right)^{n} \in 1 / \theta .
$$

Since $1 / \theta$ is a filter then, by $(10), f\left(a_{1}, \ldots, a_{k}\right) \leftrightarrow f\left(b_{1}, \ldots, b_{k}\right) \in 1 / \theta$. Thus, again by Lemma 3.4 we obtain $\left(f\left(a_{1}, \ldots, a_{k}\right), f\left(b_{1}, \ldots, b_{k}\right)\right) \in \theta$. Therefore, $f$ is $\mathcal{P O C}$-compatible.

Let $f: A^{k} \rightarrow A$ be a $\mathcal{P O C}$-compatible function, $a=\left(a_{1}, \ldots, a_{k}\right)$ and $b=$ $\left(b_{1}, \ldots, b_{k}\right)$ elements of $A^{k}$. Consider $n(a, b)$ as a natural number associated to $(a, b)$ in the Proposition 3.7. If $B$ is a finite subset of $A^{k}$ and $a \in B$ is fixed, we have a finite family of natural numbers, namely, $\{n(a, b): b \in B\}$. Let $n_{a}$ be the maximum of this family. For every $b \in B$ we define

$$
T_{b}=\left\{\left(a_{1} \leftrightarrow b_{1}\right)^{n_{a}} \cdot\left(a_{2} \leftrightarrow b_{2}\right)^{n_{a}} \cdot \ldots \cdot\left(a_{k} \leftrightarrow b_{k}\right)^{n_{a}} \cdot f(a): a \in B\right\} .
$$

The following theorem shows that every $\mathcal{P O C}$-compatible function on finite subsets can be written as a join of certain elements. Its proof is a simple adaptation of the one given in [8, Theorem 12].

Theorem 3.8. Let $f: A^{k} \rightarrow A$ be a $\mathcal{P O C}$-compatible function, $B$ a finite subset of $A^{k}$ and $b \in B$. Then there exists the supremum of $T_{b}$ and $f(b)=$ $\bigvee T_{b}$.

Proof. It follows from Proposition 3.7 that $f(b)$ is an upper bound of $T_{b}$. Since $f(b) \in T_{b}$ then $\bigvee T_{b}$ exists and $f(b)=\bigvee T_{b}$.

Let $(A, \cdot, \rightarrow, 1) \in \mathcal{P O C}$. If $(A, \leq)$ is a meet semilattice, then the pocrim is said to be a pocrim meet semilattice. The notion of pocrim join semilattice is dually defined. If $(A, \leq)$ is a lattice then the pocrim is said to be an integral commutative residuated lattice. To be more precise, a pocrim meet semilattice is an algebra $(A, \wedge, \cdot, \rightarrow, 1)$ of type $(2,2,2,0)$, a pocrim join semilattice is an algebra $(A, \vee, \cdot, \rightarrow, 1)$ of type $(2,2,2,0)$ and an integral commutative residuated lattice is an algebra $(A, \wedge, \vee, \cdot, \rightarrow, 1)$ of type $(2,2,2,2,0)$. Let us emphasize that - is a commutative operation and 1 is the greatest element respect to the order. The class of pocrim join semilattices is a variety [11, Theorem 7.1.10] and the class of pocrim meet semilattices is also a variety [11, Remark 7.1.11]. In particular, the class of integral commutative residuated lattices is a variety. We write $\mathcal{M}$ for the variety of pocrims meet 
semilattices, $\mathcal{J}$ for the variety of pocrim join semilattices and $\mathcal{R} \mathcal{L}$ for the variety of integral commutative residuated lattices.

The following proposition is a consequence of the results of this section.

Proposition 3.9. Let $\boldsymbol{A}$ be an algebra of $\mathcal{M}, \mathcal{J}$ or $\mathcal{R} \mathcal{L}$.

(1) Let $a, b \in A$. Then $(c, d) \in \theta(a, b)$ if and only if $(a \leftrightarrow b)^{n} \leq c \leftrightarrow d$ for some natural number $n$.

(2) Let $f: A^{k} \rightarrow A$ be a function. Then $f$ is compatible if and only if for every $a_{1}, \ldots, a_{k}, b_{1}, \ldots, b_{k} \in A$ there exists a natural number $n$ such that

$$
\left(a_{1} \leftrightarrow b_{1}\right)^{n} \cdot \ldots \cdot\left(a_{k} \leftrightarrow b_{k}\right)^{n} \leq f\left(a_{1}, \ldots, a_{k}\right) \leftrightarrow f\left(b_{1}, \ldots, b_{k}\right) .
$$

(3) The varieties $\mathcal{J}$ and $\mathcal{R} \mathcal{L}$ are locally affine complete, which means that every compatible function is a polynomial function over finite sets [26].

Remark 3.10. For the case of integral commutative residuated lattices the results of Proposition 3.9 are also particular cases from [8, Lemma 7], [8, Theorem 8] and [8, Corollary 13], respectively.

In this section we extended the results of Section 2 to the setting of pocrims. The main result of this section, Proposition 3.7, gives a characterization of relative compatible operations on a pocrim $\mathbf{A}$ in terms of the (monoidal) product of elements of the form $\left(a_{i} \leftrightarrow b_{i}\right)^{n}$. This result is extended to pocrim join semilattices, pocrim meet semilattices and commutative integral residuated lattices in Proposition 3.9.

For the case of pocrim meet semilattice, the unary case of Proposition 3.9 can be connected with some known results of the existing literature. In order to make explicit it, let $\mathbf{A} \in \mathcal{M}$. We define the binary term $s$ by $s(a, b):=(a \rightarrow b) \wedge(b \rightarrow a)$. Similar ideas used in lemmas 3.5 and 3.6 can be adapted in order to show the following results: 1) $(c, d) \in \theta(a, b)$ if and only if $s(a, b)^{n} \leq s(c, d)$ for some natural number $n$; 2) if $f: A \rightarrow A$ is a function, then $f$ is compatible if and only if for every $a, b \in A$ there exists a natural number $n$ such that $s(a, b)^{n} \leq s(f(a), f(b))$. In [1] Agliano covers similar territory about pocrim meet semilattices in a more general way. More precisely, part of the paper [1] is concerned with BCI-monoids and its relation with principal congruences (pocrim meet semilattices are particular cases of BCI-monoids). For example, the items 1) and 2) previosly mentioned appears in [1, pp. 409-410] for the case of BCI-monoids. Further connections with [1] may be similarly established.

\section{Appendix}

Finally we extend some of the obtained results to the cases of pseudo $B C K$ algebras and porims [27]. The techniques employed here are similar to that 
used in the previous sections, except by some facts of a combinatorial character. To understand this situation see for instance the difference between the results for compatible operations given in [8] and [9].

Definition 4.1. A pseudo $B C K$-algebra is a structure $(A, \leq, \rightarrow, \rightsquigarrow, 1)$ where $(A, \leq)$ is a poset with a greatest element 1 , and $\rightarrow, \rightsquigarrow$ are binary operations on $A$ such that for all $a, b, c \in A$ :

(PBCK1) $a \rightarrow b \leq(b \rightarrow c) \rightsquigarrow(a \rightarrow c), a \rightsquigarrow b \leq(b \rightsquigarrow c) \rightarrow(a \rightsquigarrow c)$,

$(\mathrm{PBCK} 2) a \leq(a \rightarrow b) \rightsquigarrow b, a \leq(a \rightsquigarrow b) \rightarrow b$,

(PBCK3) $a \leq b$ if and only if $a \rightarrow b=1$ if and only if $a \rightsquigarrow b=1$.

Remark 4.2. The underlying order $\leq$ can be retrieved via the condition (PBCK3). In the rest of the paper a pseudo $B C K$-algebra $(A, \leq, \rightarrow, \rightsquigarrow, 1)$ is going to be considered as an algebra $(A, \rightarrow, \rightsquigarrow, 1)$ of type $(2,2,0)$. Then it is immediate that a pseudo $B C K$-algebra is a $B C K$-algebra if and only if $\rightarrow$ and $\rightsquigarrow$ coincide.

The class of pseudo $B C K$-algebras form a quasivariety denoted by $\mathcal{P B C}$. Let $\mathbf{A} \in \mathcal{P B C K}$. Analogously to the case of $\mathcal{B C K}$, we say that $F \subseteq A$ is an implicative filter if $1 \in F$, and $b \in F$ whenever $a \in F$ and $a \rightarrow b \in F$. If $X \subseteq A$ we also write $\langle X\rangle$ for the implicative filter generated by $X$. If $X \neq \emptyset$ then $\langle X\rangle$ is described as in (2) of Section 2. In particular, the implicative filter generated by the singleton $\{a\}$ is described as in (3) of Section 2 . We say that $F$ is a compatible implicative filter if it is an implicative filter such that $a \rightarrow b \in F$ if and only if $a \rightsquigarrow b \in F$, for every $a, b \in A$. For any implicative filter $F$ of a pseudo $B C K$-algebra, the binary relation $\Theta_{F}$ is defined as in (1) of Section 2. The following result appears in [27, Proposition 2.2.4].

Lemma 4.3. Let $\boldsymbol{A} \in \mathcal{P B C K}$. There exists an order isomorphism between the poset $\operatorname{Con}_{\mathcal{B C C K}}(\boldsymbol{A})$ and the poset of compatible implicative filters of $\boldsymbol{A}$, which is established via the functions $\theta \mapsto 1 / \theta$ and $F \mapsto \Theta_{F}$.

Given $\mathbf{A} \in \mathcal{P B C K}$ and $a \in A$, by defining the polynomial functions $\rho_{a}$ and $\lambda_{a}$ as $\rho_{a}(b)=(b \rightarrow a) \rightarrow a$ and $\lambda_{a}(b)=(b \rightsquigarrow a) \rightsquigarrow a$, one can consider for every not empty $X \subseteq A$, the set

$$
\Gamma(X)=\left\{\left(\gamma_{a_{1}} \circ \cdots \circ \gamma_{a_{n}}\right)(b): b \in X, a_{i} \in A, \gamma_{a_{i}} \in\left\{\lambda_{a_{i}}, \rho_{a_{i}}\right\}, n \in \mathbb{N}\right\},
$$

where $\mathbb{N}$ is the set of natural numbers. Let $a=\left(a_{1}, \ldots, a_{2 n}\right) \in A^{2 n}$ and consider $\alpha_{a}=\lambda_{a_{1}} \circ \rho_{a_{2}} \circ \lambda_{a_{3}} \circ \ldots \circ \rho_{a_{2 n}}$. Straightforward calculations based on the fact that $\rho_{a}(a)=\lambda_{a}(a)=a$ prove that

$$
\Gamma(X)=\left\{\alpha_{a}(b): n \in \mathbb{N}, a \in A^{2 n}, b \in X\right\} .
$$

If $X=\left\{a_{1}, \ldots, a_{n}\right\}$, we write $\Gamma\left(a_{1}, \ldots, a_{n}\right)$ to denote $\Gamma\left(\left\{a_{1}, \ldots, a_{n}\right\}\right)$. 
Observe that a reformulation of [27, Theorem $2.5(\mathrm{v})]$ gives as result that an implicative filter $F$ of $\mathbf{A}$ is compatible if and only if it is closed under $\rho_{a}$ and $\lambda_{a}$ (in the sense that $\rho_{a}(b), \lambda_{a}(b) \in F$ for all $b \in F$ and $a \in A$ ).

Remark 4.4. Let $\mathbf{A} \in \mathcal{P B C K}$ and $X \subseteq A$ with $X \neq \emptyset$. If $\langle X\rangle_{c}$ stands for the compatible implicative filter generated by $X$ then it follows from $[27$, Proposition 2.7] that $\langle X\rangle_{c}=\langle\Gamma(X)\rangle$.

Let $\mathbf{A} \in \mathcal{P B C K}$ and $X=\left\{a_{1}, \ldots, a_{n}\right\} \subseteq A$. We write $\left\langle a_{1}, \ldots, a_{n}\right\rangle_{c}$ to mean $\left\langle\left\{a_{1}, \ldots, a_{n}\right\}\right\rangle_{c}$. Let $\mathbf{A} \in \mathcal{B C K}$ and $X \subseteq A$. Since the compatible implicative filters of $\mathbf{A}$ are exactly the implicative filters of $\mathbf{A}$ then $\langle X\rangle=$ $\langle X\rangle_{c}=\langle\Gamma(X)\rangle$.

Lemma 4.5. Let $\boldsymbol{A} \in \mathcal{P B C K}, a, b \in A$ and $\theta \in \operatorname{Con}_{\mathcal{P B C K}}(\boldsymbol{A})$. Then $(a, b) \in$ $\theta$ if and only if $(a \rightarrow b, 1) \in \theta$ and $(b \rightarrow a, 1) \in \theta$.

Proof. It is consequence from a direct application of Lemma 4.3.

Lemma 4.6. Let $\boldsymbol{A} \in \mathcal{P} \mathcal{B C K}$. Then $1 / \theta_{\mathcal{P B C K}}(a, b)=\langle\Gamma(a \rightarrow b, b \rightarrow a)\rangle$ for every $a, b \in A$.

Proof. If we write $\tau$ for an arbitrary $\mathcal{P B C K}$-congruence in $\mathbf{A}$, it follows from Lemma 4.5 that $\bigcap_{(a, b) \in \tau} \tau=\bigcap_{a \rightarrow b, b \rightarrow a \in 1 / \tau} \tau$. Thus,

$$
\theta_{\mathcal{P B C K}}(a, b)=\bigcap_{a \rightarrow b, b \rightarrow a \in 1 / \tau} \tau .
$$

Hence,

$$
1 / \theta_{\mathcal{P B C K}}(a, b)=\bigcap_{a \rightarrow b, b \rightarrow a \in 1 / \tau} 1 / \tau .
$$

It follows from Lemma 4.3 that $\bigcap_{a \rightarrow b, b \rightarrow a \in 1 / \tau} 1 / \tau=\langle a \rightarrow b, b \rightarrow a\rangle_{c}$. Then, by Remark 4.4 we deduce that $1 / \theta_{\mathcal{P B C K}}(a, b)=\langle\Gamma(a \rightarrow b, b \rightarrow a)\rangle$.

The following result follows from lemmas 4.5 and 4.6.

Lemma 4.7. Let $\boldsymbol{A} \in \mathcal{P B C K}$ and $a, b \in A$. Then $(c, d) \in \theta_{\mathcal{B C K}}(a, b)$ if and only if $c \rightarrow d \in\langle\Gamma(a \rightarrow b, b \rightarrow a)\rangle$ and $d \rightarrow c \in\langle\Gamma(a \rightarrow b, b \rightarrow a)\rangle$.

Lemma 4.7 allows us to bring a characterization of the unary $\mathcal{P B C K}$ compatible functions.

Proposition 4.8. Let $\boldsymbol{A} \in \mathcal{P B C K}$ and $f: A \rightarrow A$ a function. Then $f$ is $\mathcal{P B C K}$-compatible if and only if $f(a) \rightarrow f(b) \in\langle\Gamma(a \rightarrow b, b \rightarrow a)\rangle$ for every $a, b \in A$.

We dedicate the last part of this section to study the relatively compatible operations in porims. As usual, we start with some preliminary definitions and basic results. 
Definition 4.9. A porim (partially ordered residuated integral monoid) is a structure $(A, \leq, \cdot, \rightarrow, \rightsquigarrow, 1)$ where $(A, \leq)$ is a poset, $(A, \cdot, 1)$ is a monoid whose identity 1 is the greatest element of $(A, \leq)$, and the condition $a \cdot b \leq c$ if and only if $a \leq b \rightarrow c$ if and only if $b \leq a \rightsquigarrow c$ holds for all $a, b, c \in A$. Again, $\rightarrow$ and $\rightsquigarrow$ coincide if and only if $\cdot$ is commutative (in this case we have a pocrim). Porims can be described as pseudo $B C K$-algebras with the condition (P), i.e., pseudo $B C K$-algebras expanded by a binary operation which satisfies the equation $(a \cdot b) \rightarrow c=a \rightarrow(b \rightarrow c)$.

We write $\mathcal{P O}$ for the quasivariety of porims.

Remark 4.10. If $(A, \cdot, \rightarrow, \rightsquigarrow, 1) \in \mathcal{P O}$ then the operation $\cdot$ is a compatible operation of $\mathbf{A}=(A, \rightarrow, \rightsquigarrow, 1)$. In order to show it, let $\theta$ be a $\mathcal{P B C K}$ congruence of $\mathbf{A}$ and let $(a, b),(c, d) \in \theta$. Then,

$$
a \rightarrow(c \rightarrow(b \cdot d))=b \rightarrow(d \rightarrow(b \cdot d)) .
$$

Since $(a \cdot c) \rightarrow(b \cdot d)=a \rightarrow(c \rightarrow(b \cdot d))$ and $b \rightarrow(d \rightarrow(b \cdot d))=1$ then $(a \cdot c) \rightarrow(b \cdot d) \in 1 / \theta$. Analogously, $(b \cdot d) \rightarrow(a \cdot c) \in 1 / \theta$. Since $\mathbf{A} / \theta \in \mathcal{P B C K}$ then $(a \cdot c, b \cdot d) \in \theta$. Therefore, $\cdot$ is a compatible operation of $\mathbf{A}$.

For $\mathbf{A} \in \mathcal{P O}$ and $a, b \in A$ we define $a \leftrightarrow b$ as in the case of pocrims. Thus, as a consequence of Lemma 4.5 one can also state that for every $\mathbf{A} \in \mathcal{P O}$ and $a, b \in A$ it is also true that

$$
1 / \theta_{\mathcal{P O}}(a, b)=\langle\Gamma(a \leftrightarrow b)\rangle
$$

Let $\mathbf{A} \in \mathcal{P O}$ and $y \in A$. It follows from (11) that

$$
\Gamma(b)=\left\{\alpha_{a}(b): n \in \mathbb{N}, a \in A^{2 n}\right\} .
$$

Then $c \in\langle\Gamma(b)\rangle$ if and only for some $m, n$ natural numbers there exist $u_{i} \in A^{2 n}(i=1, \cdots, m)$ such that

$$
\alpha_{u_{1}}(b) \rightarrow\left(\ldots\left(\alpha_{u_{m}}(b) \rightarrow c\right) \ldots\right)=1 .
$$

But the last equality is equivalent to

$$
\alpha_{u_{1}}(b) \cdot \ldots \cdot \alpha_{u_{m}}(b) \leq c .
$$

Summarizing, we obtain the following results.

Remark 4.11. Let $\mathbf{A} \in \mathcal{P O}$ and $a, b \in A$. Then

1) $1 / \theta_{\mathcal{P O}}(a, b)=\langle\Gamma(a \leftrightarrow b)\rangle$.

2) $c \in\langle\Gamma(b)\rangle$ if and only for some $m, n$ natural numbers there exist $u_{i} \in A^{2 n}$ $(i=1, \ldots, m)$ such that $\alpha_{u_{1}}(b) \cdot \ldots \cdot \alpha_{u_{m}}(b) \leq c$. 
We use the previous remark in order to prove the following proposition.

Proposition 4.12. Let $\boldsymbol{A} \in \mathcal{P O}$ and $a, b \in A$. Then $(c, d) \in \theta_{\mathcal{P O}}(a, b)$ if and only if for some $m, n$ natural numbers there exist $u_{1}, \ldots, u_{m} \in A^{2 n}$ such that $\alpha_{u_{1}}(a \leftrightarrow b) \cdot \ldots \cdot \alpha_{u_{m}}(a \leftrightarrow b) \leq c \leftrightarrow d$.

Proof. Let $a, b \in A$. Then it follows from Lemma 4.5 that $(c, d) \in \theta_{\mathcal{P O}}(a, b)$ if and only if $(c \rightarrow d, 1) \in \theta_{\mathcal{P O}}(a, b)$ and $(d \rightarrow c, 1) \in \theta_{\mathcal{P O}}(a, b)$, which is equivalent to $c \leftrightarrow d \in 1 / \theta_{\mathcal{P O}}(a, b)$. But $1 / \theta_{\mathcal{P O}}(a, b)=\langle\Gamma(a \leftrightarrow b)\rangle$. Then $c \leftrightarrow d \in 1 / \theta_{\mathcal{P O}}(a, b)$ if and only if for some $m$ and $n$ natural numbers there exist $u_{1}, \ldots, u_{m} \in A^{2 n}$ such that $\alpha_{u_{1}}(a \leftrightarrow b) \cdot \ldots \cdot \alpha_{u_{m}}(a \leftrightarrow b) \leq c \leftrightarrow d$, which was our aim.

Corollary 4.13. Let $\boldsymbol{A} \in \mathcal{P O}$ and $f: A \rightarrow A$ an unary function. Then $f$ is a $\mathcal{P O}$-compatible function if and only if for every $a, b \in A$ there are some $m, n$ natural numbers for which there exist $u_{1}, \ldots, u_{m} \in A^{2 n}$ such that $\alpha_{u_{1}}(a \leftrightarrow b) \cdot \ldots \cdot \alpha_{u_{m}}(a \leftrightarrow b) \leq f(a) \leftrightarrow f(b)$.

Corollary 4.13 generalizes [9, Lemma 3.3] for the case of integral residuated lattices. Hence, by taking into account Corollary 4.13 we conclude that the integral case of [9, Theorem 3.8] can be generalized for any porim.

\section{Acknowledgments}

This work was supported by CONICET [PIP 112-201501-00412] and Universidad Nacional de La Plata [11/X667]. The authors acknowledge many helpful comments from the anonymous referees, which considerably improved the presentation of this paper. Finally we also would like to thank Xavier Caicedo and Antoni Torrens for their useful comments.

\section{References}

[1] Agliano P., Ternary deductive terms in residuated structures. Acta Sci. Math. (Szeged) 68, 397-429 (2002).

[2] Blok W.J. and Pigozzi D., Algebraizable Logics. Memoirs of the A.M.S. 77 No. 396 (1989).

[3] Blok W.J. and Pigozzi D., On the structure of varieties with equationally definable principal congruences III. Algebra Universalis 32, 545-608 (1994).

[4] Blok W.J. and Raftery J.G., On the variety of BCK-algebras and its subvarieties. Algebra Universalis 33, 68-90 (1995). 
[5] Blok W.J. and Raftery J.G., Varieties of commutative residuated integral pmonoids and their residuation subreducts. Journal of Algebra 190, 280-328 (1997).

[6] Caicedo X. and Cignoli R., An algebraic approach to intuitionistic connectives. Journal of Symbolic Logic 66, No. 4, 1620-1636 (2001).

[7] Caicedo X., Implicit connectives of algebraizable logics. Studia Logica 78, No. 3, 155-170 (2004).

[8] Castiglioni J.L., Menni M. and Sagastume M., Compatible operations on commutative residuated lattices. Journal of Applied Non-Classical Logics 18, 413-425 (2008).

[9] Castiglioni J.L. and San Martín H.J., Compatible operations on residuated lattices. Studia Logica, vol. 98 (1-2), 203-222 (2011).

[10] Castiglioni J.L. and San Martín H.J., On frontal operators in Hilbert algebras. Logic Journal of the IGPL 23, No. 2, 217-234 (2015).

[11] Chajda, Halaš and Kühr, Semilattice Structures. Research and Exposition in Mathematics, vol. 30, Heldermann Verlag (2007).

[12] Cignoli R. and Torrens A., Glivenko like theorems in natural expansions of BCK-logics. Mathematical Logic Quarterly 50, No. 2, 111-125 (2004).

[13] Cornish, W.H., On Iséki's BCK-algebras. Lecture Notes in Pure and Applied Mathematics 74, 101-122, Dekker, New York (1982).

[14] Diego A., Sobre Algebras de Hilbert. Notas de Lógica Matemática. Instituto de Matemática, Universidad Nacional del Sur, Bahía Blanca, Argentina (1965).

[15] Esakia L., The modalized Heyting calculus: a conservative modal extension of the Intuitionistic Logic. Journal of Applied Non-Classical Logics vol. 16, No. 3-4, 349-366 (2006).

[16] Fried E., Grátzer G. and Quackenbush R., Uniform congruence schemes. Algebra Universalis 10, 101-122 (1980).

[17] Galatos N., Jipsen P., Kowalski T. and Ono H., Residuated lattices: an algebraic glimpse at substructural logics. Studies in Logic and the Foundations of Mathematics, vol. 151, Elsevier (2007).

[18] García Olmedo F.M. and Rodríguez Salas A.J., Linearization of the BCK-logic. Studia Logica 65, 31-51 (2000). 
[19] Gorbunov A.V., Algebraic Theory of quasivarieties. Plenum Publishing Co., New York (1998).

[20] Higgs D., Dually residuated commutative monoids with identity element as least element do not form an equational class. Math. Jap. 29, 69-75 (1989).

[21] Idziak P.M., Lattice operations in BCK-algebras. Math Japonica 29, 839-846 (1984).

[22] Idziak P.M., Filters and congruences in BCK-semilattices. Math. Japonica 29, 975-980 (1984).

[23] Iséki K., On ideals in BCK-algebras. Math. Sem. Notes Kobe Univ. 3, 1-12 (1975).

[24] Iséki K. and Tanaka S., Ideal theory of BCK-algebras. Math. Japon. 21, 351-366 (1976).

[25] Iséki K. and Tanaka S., An introduction to the theory of BCK-algebras. Math Japonica 23, 1-26 (1978).

[26] Kaarli K. and Pixley A. F., Polynomial completeness in algebraic systems. Chapman and Hall/CRC (2001).

[27] Kühr J., Pseudo BCK-algebras and related structures (Ph.D. thesis). Univerzita Palackèho v Olomouci (2007).

[28] Meng J. and Jun Y.B., BCK-algebras. Kyung Moon Sa. Co., Seoul Korea (1994).

[29] Muravitsky A.Y., Logic KM: A biography, in: Leo Esakia on Duality of Modal and Intuitionistics logics. Series: Outstanding Contributions to Logic, 4, Springer (2014), 147-177.

[30] Pixley A., Completeness in arithmetical algebras. Algebra Universalis 2, No. 1, 179-196 (1972).

[31] Simonova A.G., Interpolation property for extensions of intuitionistic proof logic. Mathematical Notes of the Academy of Sciences of the USSR. Volume 47, Issue 5, pp. 483-490 (1990).

[32] Wronski A., BCK-algebras do not form a variety. Math. Japonica 28, 211-213 (1983). 